\title{
Démodulation AM par boucle de Costas numérique
}

\author{
Hervé BOEGLEN, Laurent MURA \\ IUT de Colmar, Département R\&T, 34, rue du Grillenbreit, 68008 Colmar Cedex, France
}

herve.boeglen@uha.fr, laurent.mura@uha.fr

\begin{abstract}
RESUME : Dans cet article, nous décrivons la démarche que nous avons suivie pour concevoir une boucle de Costas entièrement numérique et que nous utilisons dans le cadre d'une séance de travaux pratiques pour démoduler un signal modulé en amplitude de façon synchrone. Nous montrons également que cette structure numérique présente l'avantage d'illustrer de manière simple et concrète à des étudiants de $2^{\text {ème }}$ année de DUT Réseaux et Télécommunications (R\&T) les notions d'évanouissement et de signal analytique.
\end{abstract}

Mots clés : Traitement numérique du signal, télécommunications.

\section{INTRODUCTION}

Dans le cadre du module de $2^{\text {ème }}$ année «Traitement Numérique du Signal » du DUT R\&T, nous sommes de plus en plus confrontés à des étudiants « allergiques » à tout nouveau concept dès lors qu'il fait appel à des équations mathématiques. La solution pédagogique à ce problème consiste à motiver les étudiants en leur présentant des systèmes de télécommunications parfaitement fonctionnels sous forme de Travaux Pratiques (TP). Lorsque cette approche pratique a été réalisée, la présentation des concepts théoriques est plus aisée. Les systèmes de télécommunication modernes sont toutefois très complexes et concevoir une démonstration pédagogique n'est généralement pas chose aisée. L'avènement des systèmes numériques, avec notamment les processeurs de traitement numérique du signal (Digital Signal Processors ou DSP) et leurs outils de développement permet de simplifier les étapes de conception d'un système de télécommunication à visée pédagogique.

L'objectif de cet article est donc d'une part, de décrire les étapes de conception d'un système de transmission numérique à visée pédagogique et d'autre part de rendre compte du résultat d'une expérience pédagogique menée en suivant cette démarche à travers l'exemple d'un système de transmission synchrone en modulation d'amplitude.

\section{PRESENTATION DU CONTEXTE}

Les TP de traitement numérique du signal sont effectués sur des maquettes C6713DSK de Texas Instruments. Ces maquettes intègrent un CODEC stéréo permettant de traiter des signaux jusqu'à une fréquence d'échantillonnage de $96 \mathrm{KHz}$ ce qui les limite à des applications audio. Les séances de TP sont au nombre de quatre. Les deux premières séances permettent d'une part, de prendre en main l'environnement de développement appelé Code Composer Studio, qui est un environnement de développement permettant de programmer le DSP en $\mathrm{C}$ ou $\mathrm{C}++$, et d'autre part, d’introduire la génération de signaux audionumériques périodiques et le filtrage numérique de type Réponse Impulsionnelle Finie (RIF) et Réponse Impulsionnelle Infinie (RII). En ce qui concerne les séances suivantes, et afin de répondre à la demande des étudiants qui réclament sans cesse des applications concrètes dans le domaine des télécommunications, nous avons élaboré un système de transmission en modulation d'amplitude à double bande latérale avec porteuse (Double Side Band Full Carrier Amplitude Modulation ou DSB-FC AM) dont le synoptique est représenté sur la Figure 1.

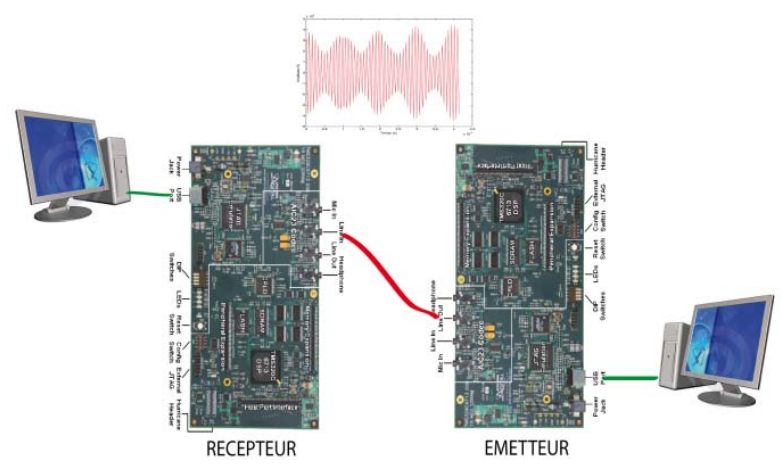

Figure 1 : Système de transmission AM à DSP

Au cours de la $3^{\text {ème }}$ séance, les étudiants travaillent en deux groupes: le $1^{\mathrm{er}}$ groupe implémente l'émission $\mathrm{AM}$ et le $2^{\text {ème }}$ groupe met en œuvre le récepteur AM. Pour l'émission, la tâche consiste d'abord à acquérir un signal audio qui ensuite module en amplitude une porteuse de fréquence $\mathrm{f}=12 \mathrm{KHz}$. Avant modulation, le 
signal est filtré passe-bas pour éviter le repliement de spectre. En ce qui concerne la réception, le travail consiste à démoduler le signal AM provenant de la carte DSP émettrice puis de le restituer sur les haut-parleurs du PC.

Dans les deux cas (émetteur et récepteur), les difficultés concernent la mise en œuvre du filtrage numérique et la réalisation d'un oscillateur numérique à l'aide d'une équation aux différences. Ces notions ont déjà été abordées lors des séances de travaux dirigés ainsi que lors des deux premières séances de $\mathrm{TP}$ ce qui fait que la majorité des étudiants parvient au cours des 4 heures de la séance à avoir un système fonctionnel. C'est à ce niveau qu'intervient la mise en évidence d'un défaut très connu dans le domaine des télécommunications : l'évanouissement du signal de réception du fait de l'absence de synchronisme entre les oscillateurs d'émission et de réception. Les étudiants constatent effectivement de manière concrète que l'amplitude du signal audio diminue cycliquement jusqu'à disparaître complètement sur une durée d'une dizaine de secondes!

Ce défaut nous a amené à concevoir une $4{ }^{\text {ème }}$ séance de TP qui consiste à mettre en œuvre un récepteur à boucle de Costas [1] entièrement numérique. La démarche de conception que nous avons suivie met en œuvre de nombreux concepts rencontrés couramment en transmission numérique. C'est la raison pour laquelle nous allons détailler ci-après les éléments qui nous paraissent les plus significatifs de cette démarche.

\section{BOUCLE DE COSTAS : PASSAGE D'UNE STRUCTURE ANALOGIQUE A UNE STRUC- TURE NUMERIQUE}

\subsection{La boucle de Costas analogique}

En parcourant la littérature, on la retrouve souvent dans le cas de la démodulation synchrone d'un signal modulé en amplitude sans porteuse (Double Sideband Suppressed Carrier ou DSB-SC). Sa structure est représentée sur la Figure 2. Il s'agit d'une boucle à verrouillage de phase (Phase-locked Loop ou PLL) d'un type particulier. Elle est constituée de deux détecteurs cohérents qui reçoivent le même signal d'entrée $\mathrm{u}(\mathrm{t})=\mathrm{b}(\mathrm{t}) \cos \left(\omega_{0} \mathrm{t}\right)$ mais avec des signaux d'oscillateurs locaux différents $\mathrm{v}_{1}(\mathrm{t})$ et $\mathrm{v}_{2}(\mathrm{t})$ qui sont en quadrature de phase. L'Oscillateur Contrôlé en Tension (OCT) est ajusté à la fréquence de la porteuse $\mathrm{f}_{0}=\omega_{0} / 2 \pi$ connue à priori. Le détecteur de la branche supérieure est défini comme le détecteur en phase (In-phase) ou canal I, celui de la branche inférieure comme le détecteur en quadrature (Quadrature-phase) ou canal Q. Ces deux détecteurs sont couplés pour former un système contre-réactionné conçu pour maintenir l'oscillateur local en synchronisme avec la porteuse. Pour de plus amples détails sur cette structure, on pourra se référer aux références [1] et [2].

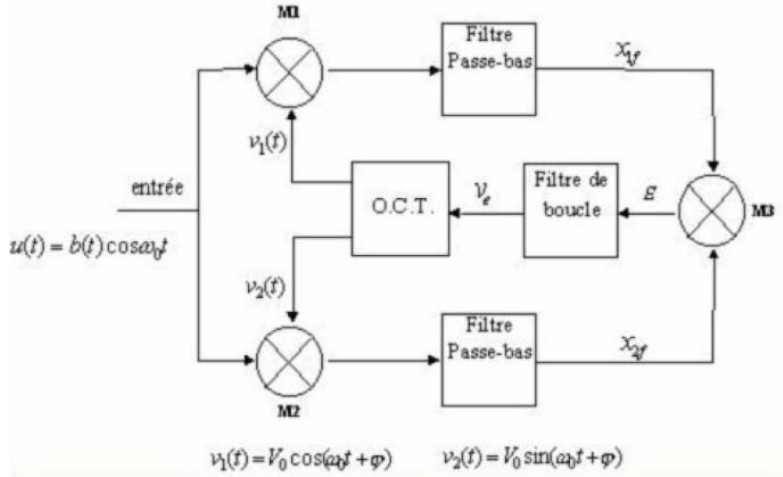

Figure 2 : Boucle de Costas analogique

La conversion de cette structure analogique en une structure numérique fonctionnant en temps réel sur un DSP pose le problème du nombre de filtres. En plus du filtre de boucle, on est en présence de deux filtres passe-bas. L'opération de filtrage numérique étant " gourmande » en temps de calcul on va donc opter pour une structure plus adaptée que nous présentons dans le paragraphe suivant.

\subsection{La boucle de Costas numérique}

Contrairement à la structure analogique, on a beaucoup plus de difficultés à trouver des informations pertinentes permettant la mise en œuvre de la boucle de Costas numérique. La référence [3] propose la structure représentée sur la Figure 3.

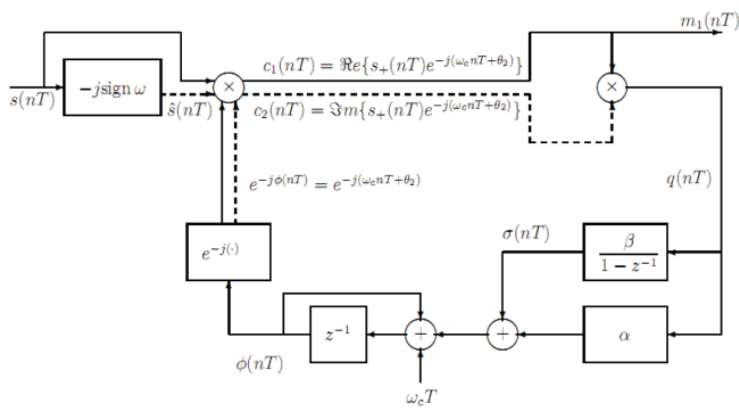

Figure 3 : Boucle de Costas numérique

Il s'agit d'une boucle d'ordre 2, le filtre de boucle analogique ayant été converti en son équivalent numérique par transformation bilinéaire. Le lecteur intéressé par le détail de la conversion d'une PLL analogique en PLL numérique pourra consulter avec bonheur les deux excellentes références [4] et [5]. Sans perte de généralité, supposons que le signal d'entrée soit égal à :

$$
\mathrm{s}(\mathrm{nT})=\mathrm{A}_{\mathrm{c}} \mathrm{m}(\mathrm{nT}) \cos \left(\omega_{\mathrm{c}} \mathrm{nT}+\theta_{1}\right)
$$

où $\omega_{\mathrm{c}}$ est la pulsation de la porteuse et $\mathrm{T}$ représente le temps d'échantillonnage du système. S’il y a un décalage de fréquence, provoqué par exemple par un mauvais alignement des fréquences des porteuses d'émission et de réception, on aura :

$$
\theta_{1}=\Delta \omega n T+\gamma
$$


où $\Delta \omega$ représente un décalage de fréquence entre l'émission et la réception et $\gamma$ un offset de phase constant.

La première étape du système consiste à former l'enveloppe complexe du signal d'entrée :

$$
\mathrm{S}_{+}(\mathrm{nT})=\mathrm{s}(\mathrm{nT})+\mathrm{j} \hat{s}(\mathrm{nT})=\mathrm{A}_{\mathrm{c}} \mathrm{m}(\mathrm{nT}) e^{j\left(\omega_{c} n T+\theta_{1}\right)}
$$

Les lignes parallèles en trait plein et en pointillés sur la Figure 3 représentent des signaux complexes : les traits pleins correspondants à la partie réelle et les traits en pointillés à la partie imaginaire. Le système génère l'estimation $\Phi(\mathrm{nT})$ de l'angle du signal reçu qui s'écrit :

$$
\Phi(\mathrm{nT})=\omega_{\mathrm{c}} \mathrm{nT}+\theta_{2}
$$

La boucle est verrouillée lorsque l'erreur de phase $\theta_{1}$ $\theta_{2}$ reste faible. Lorsqu'elle est nulle, le signal démodulé apparaît au point $\mathrm{c}_{1}(\mathrm{nT})=\mathrm{m}_{1}(\mathrm{nT})$ et $\mathrm{c}_{2}(\mathrm{nT})=0$.

\subsection{Implémentation de la boucle de Costas numé- rique}

Nous décrivons maintenant la démarche que nous avons suivie pour implémenter la boucle de Costas numérique.

\subsubsection{Utilisation de l'outil Simulink}

Cet outil, très connu dans la communauté du traitement du signal, permet de se rendre compte rapidement du fonctionnement général d'une structure représentée sous la forme de schéma-blocs. Avant de présenter le schéma complet, nous allons insister sur deux éléments qui nous paraissent représentatifs d'une implémentation numérique, à savoir un filtre de Hilbert numérique et un oscillateur contrôlé numériquement.

Le premier élément concerne la génération de l'enveloppe complexe à partir du signal s(nT). Pour créer $\hat{s}(\mathrm{nT}), \mathrm{s}(\mathrm{nT})$ doit être appliqué à l'entrée d'un système qui implémente la transformée de Hilbert [6]. La réponse impulsionnelle d'un filtre de Hilbert en temps continu est donnée par :

$$
h(t)=\frac{1}{\pi t}
$$

Sa réponse fréquentielle est définie de la manière suivante :

$$
H(j \omega)=\left\{\begin{array}{l}
-j \text { pour } \omega>0 \\
0 \text { pour } \omega=0 \\
+j \text { pour } \omega<0
\end{array}\right.
$$

Le gain de la réponse fréquentielle est unitaire (à l'exception du $0 \mathrm{~Hz}$ ) et le filtre introduit un déphasage de $-90^{\circ}$ pour les fréquences positives et un déphasage de $+90^{\circ}$ pour les fréquences négatives. Ce filtre analogique peut être approximé par un filtre numérique de type RIF ou par une opération de type «FFT/Déphasage/IFFT ». Dans notre cas, nous avons choisi l'approche RIF. Les coefficients de ce filtre sont obtenus pas le commande firpm de Matlab (algorithme de Parks-McClellan). Pour un filtre de 30 coefficients, la commande s’écrit :

$B=\operatorname{firpm}\left(30,\left[\begin{array}{ll}.1 & .9\end{array}\right],\left[\begin{array}{ll}1 & 1\end{array}\right]\right.$, 'Hilbert');

Ce filtre introduit un retard de 15 échantillons qu'il faut penser à compenser. La Figure 4 donne le schémablocs Simulink que nous avons obtenu pour valider cette structure.

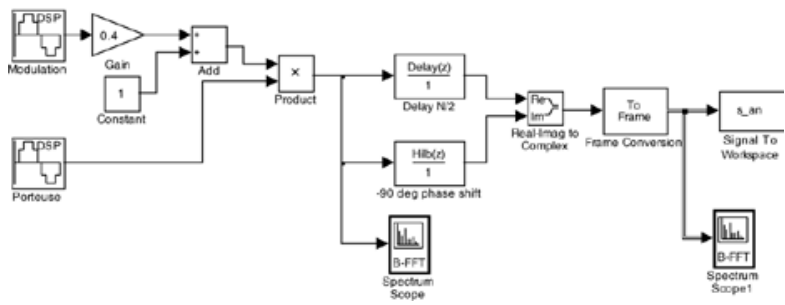

Figure 4 : Test du filtrage de Hilbert

Nous générons un signal AM DSB-FC que nous passons dans le filtre de Hilbert numérique. Le résultat obtenu est conforme à notre cahier des charges et est présenté sur la Figure 5.

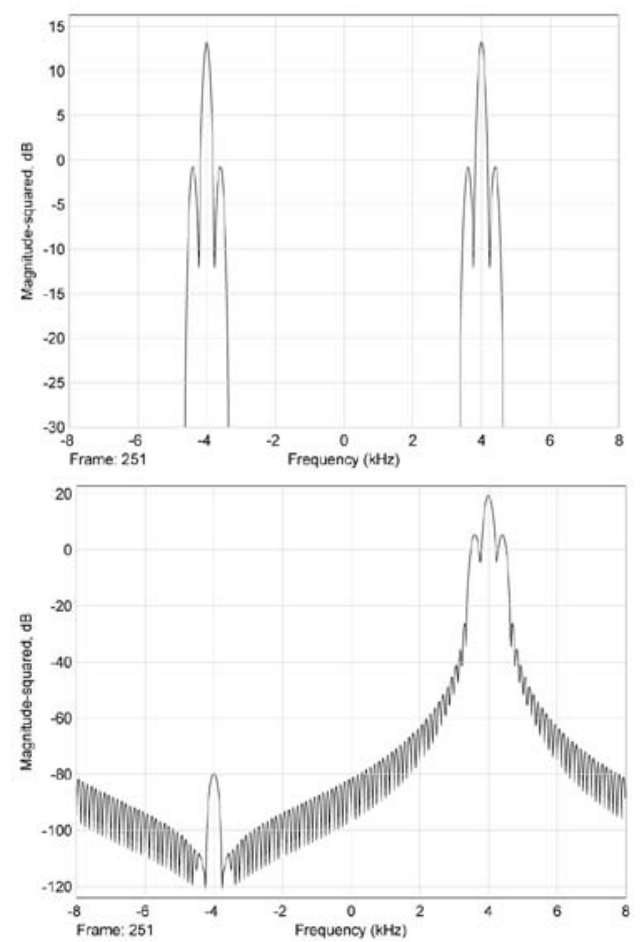

Figure 5 : Signal AM DSB-FC avant (haut) et après (bas) filtrage de Hilbert.

Voyons maintenant comment réaliser l'équivalent de l'OCT de la structure analogique. On parlera alors d'un oscillateur contrôlé numériquement (Numerically Controlled Oscillator ou NCO). Le modèle Simulink cor- 
respondant est représenté sur la Figure 6. Il s’agit d'une structure de type Synthèse Numérique Directe (Direct Digital Synthesis ou DDS) qui permet à partir d'un accumulateur de phase modulo $2 \pi$ de ǵnérer les $\mathrm{v}$ aleurs de cosinus et de sinus à la fréquence souhaitée.

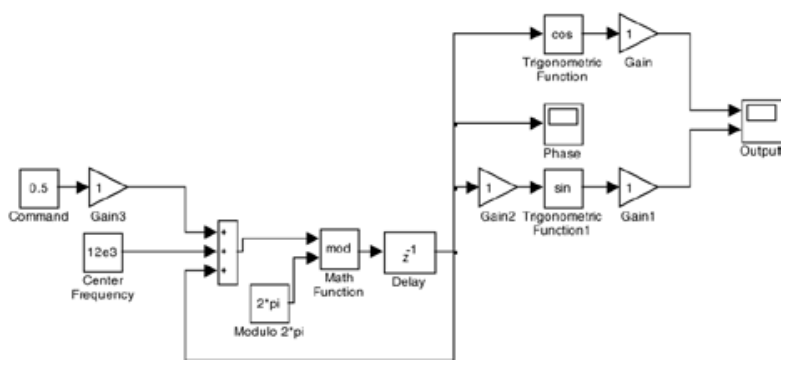

Figure 6 : Structure du NCO.

En fonction de la valeur de la commande, on peut faire varier la fréquence du système autour de la fréquence centrale (Center Frequency sur la Figure 6) [5]. La Figure 7 donne un exemple de simulation. Le graphe du haut de la figure montre l'évolution de l'accumulateur de phase (sortie Phase sur la Figure 6) et le graphe du bas la sortie du DDS (sortie Output sur la Figure 6).
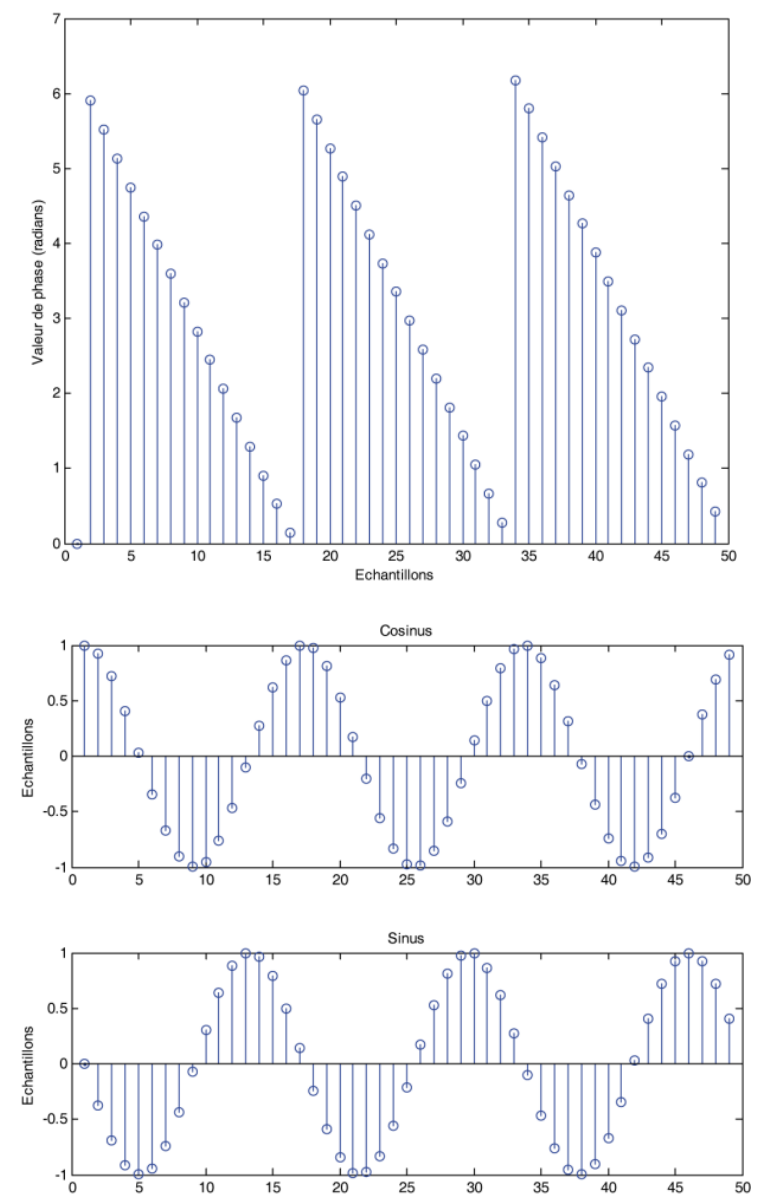

Figure 7 : NCO : exemple de simulation.
Nous disposons maintenant des éléments essentiels au fonctionnement de la boucle de Costas numérique. Le schéma complet est représenté sur la Figure 8 de la page suivante. La structure a été testée avec succès en démodulant un signal AM DSB-FC qui nécessite de rajouter un filtre passe-haut pour éliminer la composante continue résiduelle (cf. Figure 8).

\subsubsection{Du schéma-blocs au code Matlab}

L’implémentation Simulink sous forme de schémablocs permet de bien saisir le fonctionnement d'un système numérique. Cependant, son implémentation sur DSP nécessite de disposer d'un code informatique en langage $\mathrm{C}$ ou $\mathrm{C}++$. Bien que The Mathworks propose la possibilité de passer d'un modèle Simulink à un code $\mathrm{C}$ en quelques clics de souris, nous n'avons pas choisit cette voie. En effet, hormis le coût relativement élevé des boîtes à outils spécifiques à rajouter, le code généré n'est pas très lisible et n'est pas suffisamment concis pour une exploitation en temps réel. Notre approche a donc consisté à traduire le modèle Simulink en code Matlab très proche du langage C. La Figure 9 donne un extrait de ce code.

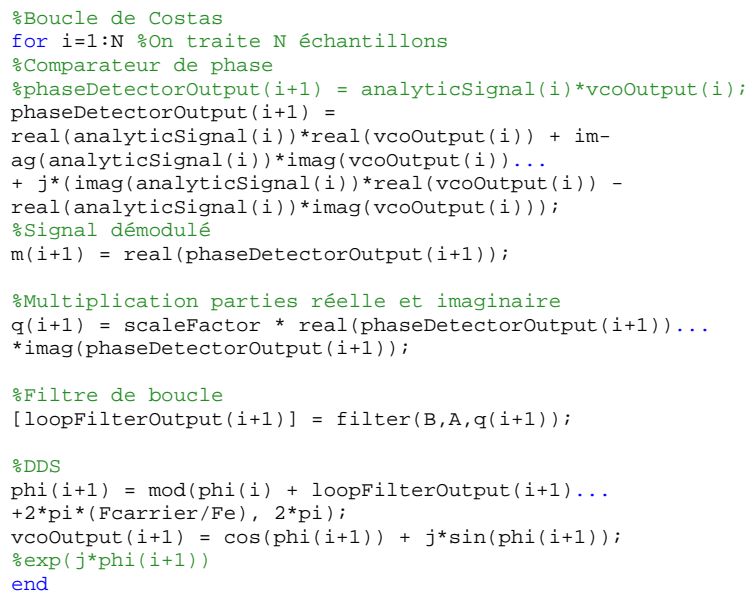

Figure 9 : Extrait du code Matlab réalisant la boucle de Costas.

3.3.3.

Implémentation sur le DSP

Le code Matlab étant très proche d'un code $C$, cette dernière étape a été une formalité. La seule difficulté consiste à avoir une base de temps fiable qui est obtenue à partir de l'horloge du CODEC intégré à la carte de développement C6713DSK. Le code de la boucle est alors placé dans une routine d'interruption appelée régulièrement au rythme de l'horloge du CODEC.

\section{MISE EN EUUVRE LORS D'UNE SEANCE DE TRAVAUX PRATIQUES}

On conçoit que la mise en œuvre d'une structure comme la boucle de Costas numérique ne se fait pas en quelques minutes ni même en quatre heures (la durée 


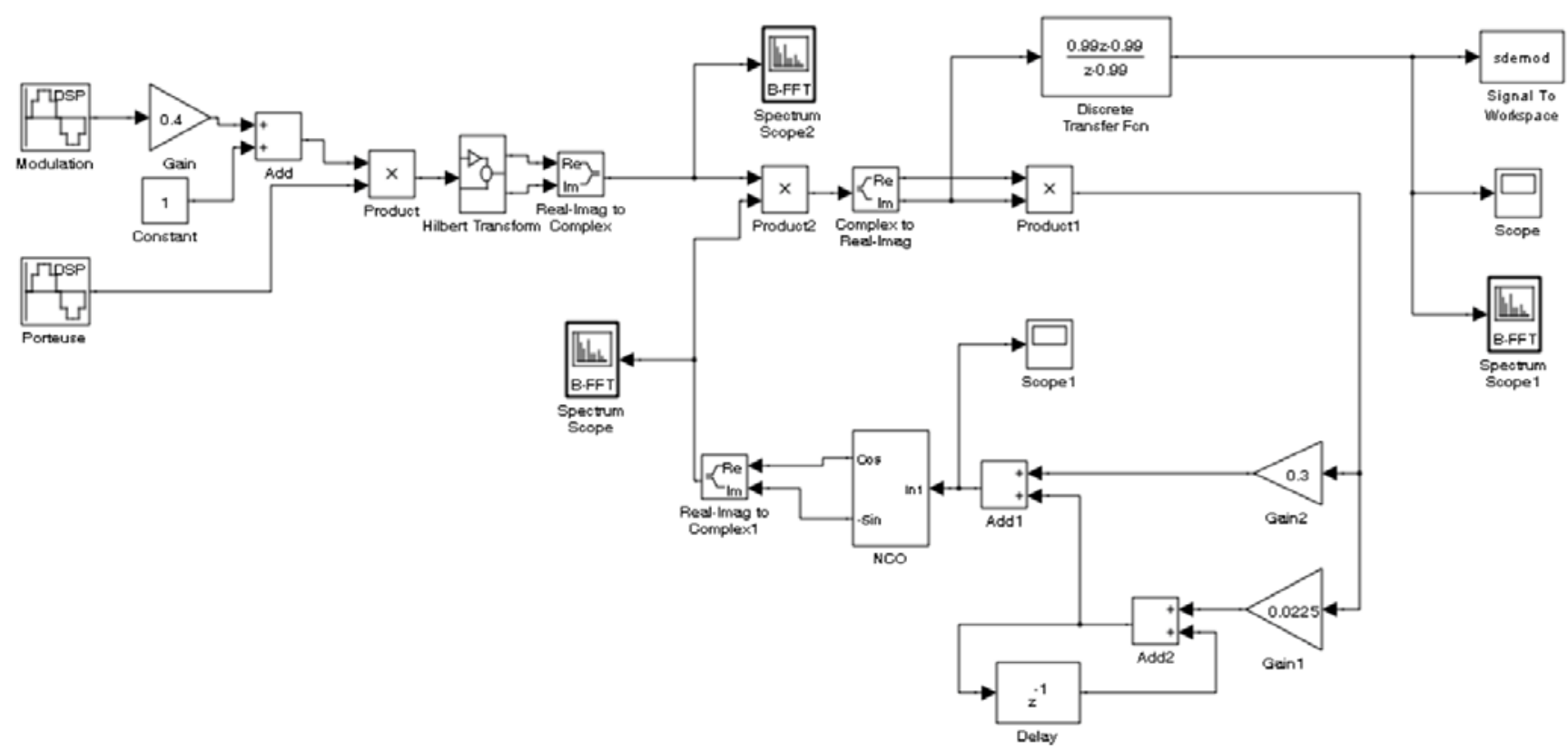

Figure 8 : Schéma-blocs de la boucle de Costas.

de la séance de TP). Nous donnons donc aux étudiants l'ensemble du projet informatique en omettant deux lignes qu'il faut compléter. Ces lignes concernent le filtre de Hilbert qu'ils doivent étudier et implémenter préalablement sous Matlab et le NCO qu'ils ont déjà rencontré lors des séances de travaux dirigés. Cela ne pose pas de problème particulier, puisque la majorité des étudiants parvient à tester le système. Pour éprouver sa robustesse, il leur est également demandé d'introduire des décalages fréquentiels de quelques centaines de Hertz au niveau de la porteuse d'émission pour observer le comportement de la boucle. Cette étape est vraiment parlante car on entend effectivement un sifflement qui se rajoute au signal audio lorsque la boucle compense le décalage fréquentiel !

\section{CONCLUSION}

Nous venons de présenter la démarche qui nous a permis de concevoir une boucle de Costas entièrement numérique. Contrairement à l'approche analogique qui consiste à mettre au point une carte électronique (cf. la référence [2]), la conception d'un système de télécommunication numérique fait appel aux notions issues du traitement numérique du signal qui sont aisément mises en œuvre à l'aide d'un ordinateur associé à une carte à DSP et à des outils logiciels. De plus, la mise au point du système est aisée, nul besoin de fer à souder, il suffit d'ajuster les paramètres et de recompiler et le tour est joué !

Ce système a été conçu pour corriger un défaut constaté dans un TP de transmission en modulation AM. Il permet aux étudiants de mettre en œuvre un système concret couramment rencontré en pratique. Sa mise en œuvre a été un réel succès auprès de nos étudiants de $2^{\text {ème }}$ année de DUT R\&T.
Enfin, à nouvelles pratiques de conception, nouvelles méthodes de partage des expériences. Après une démonstration du système lors du colloque CETSIS 2010, l'ensemble des fichiers informatiques sera mis à disposition de la communauté sur le site web de l'un des auteurs [7] !

\section{Bibliographie}

[1] S. Haykin, "Communication systems", $4^{\text {th }}$ edition, Wiley, 2001.

[2] C. Chatellier, J. Brochard, "Récupération de porteuse dans les systèmes de transmissions analogiques et numériques”, J3eA, Vol. 3 (2004) 5.

[3] D.R. Stephens, "Communication system design using DSP algorithms", Springer, 2008.

[4] S. A. Tretter, "Phase-locked loops for wireless communications”, $2^{\text {nd }}$ Edition, Kluwer Academic Publisher, 2002.

[5] M. Rice, "Digital communications: a Discrete-Time approach", Prentice Hall, 2008.

[6] G. Blanchet, M. Charbit, "Signaux et images sous Matlab”, Hermes Science, 2001.

[7] http://herve.boeglen.free.fr/cetsis2010 